\title{
A Flexible State of Health Prediction Scheme for Lithium-Ion Battery Packs with Long Short- Term Memory Network and Transfer Learning
}

\author{
Xing Shu, Graduate Student Member, IEEE, Jiangwei Shen, Guang Li, Senior Member, IEEE, \\ Yuanjian Zhang, Member, IEEE, Zheng Chen, Senior Member, IEEE and Yonggang Liu, Senior \\ Member, IEEE
}

\begin{abstract}
The application of machine learning-based state of health (SOH) prediction is hindered by large demand of training data. To conquer this defect, a flexible and easy transferred SOH prediction scheme for lithium-ion battery packs is developed. Firstly, the charging duration for a predefined voltage range is hired as the health feature to quantify capacity degradation. Then, the long short-term memory (LSTM) network and transfer learning (TL) with fine-tuning strategy are incorporated to constitute the cell mean model (CMM) for SOH prediction with partial training data. Next, to evaluate the $\mathrm{SOH}$ inconsistencies among cells, the LSTM model is employed as the cell difference model (CDM), and the minimum estimation value of CDM is identified to determine pack $\mathrm{SOH}$. The experimental results reveal that even when the first 360 cycle data, occupying only $40 \%$ in the whole 904 cycle data, are chosen and constituted to the dataset for model training, the obtained estimation algorithm can still predict SOH precisely with the error of less than $3 \%$, thus remarkably reducing the training data amount and mitigating the computation burden during model training. In addition, the preferable validation results on different types of lithium-ion batteries further manifest the extendibility of the proposed strategy.
\end{abstract}

Index Terms-Lithium-ion battery pack, long short-term memory (LSTM), transfer learning (TL), state of health (SOH).

\section{INTRODUCTION}

$\mathrm{D}$ UE to the massive consumption of fossil fuel and pressing concerns over carbon emissions, high-efficiency and environment-friendly electric vehicles (EVs) have become the development mainstream in transportation electrification [1,2]. In EVs, lithium-ion batteries have been widely deployed as main energy sources, with a form of pack consisting of tens to thousands of cells connected in series, parallel or mixed topologies [3, 4]. However, with the increase of operation, battery performance degrades gradually, including capacity

This work was supported in part by the National Key R\&D Program of China (No. 2018YFB0104000), in part by the National Natural Science Foundation of China (No. 61763021), and in part by the EU-funded Marie Skłodowska-Curie Individual Fellowships (No. 845102). (Corresponding Authors: Zheng Chen and Yonggang Liu)

Xing Shu and Jiangwei Shen are with the Faculty of Transportation Engineering, Kunming University of Science and Technology, Kunming, 650500, China (e-mail: shuxing92@ kust.edu.cn; shenjiangwei6@163.com).

Guang Li is with the School of Engineering and Materials Science, Queen Mary University of London, London, E1 4NS, United Kingdom (e-mail: g.li@qmul.ac.uk). decline, resistance increase and inconsistency aggravation among cells [5]. It is, therefore, critical to reliably estimate the healthy status of lithium-ion batteries, referred to as state of health $(\mathrm{SOH})$ prediction [6]. Accurate knowledge of $\mathrm{SOH}$ cannot only contribute to the estimation promotion of state of charge (SOC), but also facilitate safe operation of batteries [7].

Battery $\mathrm{SOH}$ represents the ratio of maximum discharging capacity over the rated value [8]. Generally, the prediction methods of $\mathrm{SOH}$ can be sorted into three groups: direct calibration methods, filter-based methods and machine learning-based methods [9]. Direct calibration methods determine battery $\mathrm{SOH}$ via specific experimental operations, such as full discharge of the battery after a complete charge [10]. This kind of methods are simple and easy to implement. Nevertheless, it is an open-loop approach highly depending on the acquisition accuracy of current and voltage; and furthermore it is impractical to fully charge and discharge the batteries equipped in EVs. To cope with the restrictions of direct calibration methods, filter-based methods are developed, and they treat $\mathrm{SOH}$ as one parameter waiting to be identified in the model or one state variable that needs to be estimated in the built observer [11]. Ordinary filter algorithms, such as extended Kalman filter (EKF) [12], particle filter [13] and H-infinity filter [14], have been intensively applied for online $\mathrm{SOH}$ estimation. However, the performance of these methods highly relies on the accuracy and robustness of the established battery model or observer.

Machine learning-based methods have been progressively exploited to predict $\mathrm{SOH}$ due to their strong data processing and nonlinear fitting capabilities [15]. These methods usually employ different machine learning algorithms to capture the degradation trend from measurement and generate healthy features representing SOH variation [16]. Ref. [17] reveals that

Yuanjian Zhang is with the School of Mechanical and Aerospace Engineering, Queen's University of Belfast, BT9 5AG, Northern Ireland. (y.zhang@qub.ac.uk).

Zheng Chen is with the Faculty of Transportation Engineering, Kunming University of Science and Technology, Kunming, 650500, China and also with the School of Engineering and Materials Science, Queen Mary University of London, London E1 4NS, U.K. (e-mail: chen@kust.edu.cn).

Yonggang Liu is with the State Key Laboratory of Mechanical Transmissions \& College of Mechanical and Vehicle Engineering, Chongqing University, Chongqing 400044, China (e-mail: andylyg@umich.edu). 
the partial differential temperature variation is related to $\mathrm{SOH}$ degradation, and then the support vector regression (SVR) is exploited to predict $\mathrm{SOH}$ based on temperature variation. In [18], Gaussian process regression (GPR) is introduced to map the relationship between partial capacity increase and SOH. In addition to SVR and GPR methods, random forest regression (RFR) [19], radial basis function neural network (RBFNN) [20], relevance vector machine (RVM) [21] and long short-term memory (LSTM) network [22] are also successfully leveraged to estimate SOH. Among these machine learning algorithms, LSTM network, as a typical representative of recurrent neural networks (RNNs), can capture the long-term dependency information through its specially designed gate structure, and overcome the drawbacks of gradient explosion and gradient vanishing existing in RNN. Consequently, LSTM network is widely adopted in state prediction due to its superior performance in capturing long-term relationship of historical information [23].

Although the above-mentioned approaches can achieve satisfactory $\mathrm{SOH}$ prediction, a large amount of training data are indispensable, undoubtedly increasing the duration of offline test. Moreover, when the battery type or the health feature is changed, the pre-determined model needs to be reconstructed, or the model parameters need to be retrained, obviously complicating the algorithm design. Ref. [24] exerts the GPR to estimate $\mathrm{SOH}$ based on two different types of batteries. However, when the GPR model trained on the basis of dataset 1 is transplanted to estimate the battery $\mathrm{SOH}$ described in dataset 2 , the repetitive operations, including reselecting the model parameters and retraining the model, need to be conducted once again. The similar difficulties are also encountered in [19], where the relative incremental capacity within three different voltage ranges are considered as healthy features to characterize the $\mathrm{SOH}$ variation. However, the built model needs to be retrained with massive cycle data after the voltage range varies. In real-time applications, it is timeconsuming, material-consuming and labor-intensive to do so. How to mitigate the burdensome training job or reduce the required data amount when constructing the $\mathrm{SOH}$ model based on machine learning algorithms needs to be further investigated.

Another critical concern for $\mathrm{SOH}$ estimation is the inconsistency among battery cells, which is ineluctable in production and usage, especially for EV application. To the author's knowledge, there is still lack of efficient manners to characterize the SOH of battery packs on the basis of cell's $\mathrm{SOH}$. To diagnose the health state of lithium-ion battery packs, Ref. [25] applies a multi-stage constant current (CC) charging strategy to accelerate battery pack degradation, and a dual GPR framework is advanced to simultaneously forecast $\mathrm{SOH}$ and remaining useful life (RUL). However, the whole pack is deemed as an integrated unit without considering the inconsistency among cells. In [26], to identify the inconsistency of cell SOC, a cell mean model (CMM) and a cell difference model $(\mathrm{CDM})$ are respectively constructed to delineate the overall SOC of pack and the difference between cell SOC and mean value. Nevertheless, few methods have been devoted to the estimation of cell SOH inconsistency in battery packs. It is, therefore, imperative to design a straightforward and efficient pack $\mathrm{SOH}$ estimation method to fill in the gap of existing research.

Aiming to promote the potential of machine learning-based methods and investigate an efficient pack $\mathrm{SOH}$ prediction algorithm, a flexible and transferable $\mathrm{SOH}$ prediction scheme is advanced for lithium-ion battery packs. Concretely, a LSTM network integrating a fully connected layer is constructed to learn the long-term dependency of battery cell SOH according to the features generated from partial charging voltage data. On this basis, a transfer learning (TL) method, which is applied to improve the learning ability by transferring information from a related domain, is proposed for easy adaption to the variation of feature voltages existing in different batteries, thereby significantly mitigating the burdensome training task. In addition, to fully consider the inconsistency among battery cells, the CMM and CDM are respectively addressed to simulate mean $\mathrm{SOH}$ level of pack and difference of cell $\mathrm{SOH}$, thereby efficiently identifying the pack SOH. Eight battery cells and three types of batteries are experimented to verify the effectiveness of the proposed method. In addition, more indepth validations on another two different types of lithium-ion batteries further justify the extendibility and flexibility of the proposed algorithm.

The reminder of this paper is structured as follows: the main methodologies including LSTM and TL are introduced in Section II. The experimental tests and generation process of health features are described in Section III. Next, the detailed prediction procedure of pack $\mathrm{SOH}$ is detailed in Section IV. After that, Section $\mathrm{V}$ analyzes and discusses the experimental validation results. Finally, Section VI draws the main conclusions.

\section{MethodOLOGIES}

The objective of this study is to construct a uniform scheme to forecast SOH with partial training data based on LSTM and TL. To attain it, the theoretical basis of both algorithms are detailed first.

\section{A. Long Short-Term Memory Network}

The LSTM network, as an extended form of RNN, exploits the memory units in place of conventional hidden nodes to avert gradient vanishing or explosion [27]. Fig. 1 exhibits the schematic diagram of LSTM, which includes an input gate, an output gate and a forget gate [28, 29]. They are in charge of receiving data, outputting estimation and eliminating unnecessary information, respectively. Another key part of LSTM is the state cell, of which the main function is to store a summary of previous input sequences. Additionally, the structure of LSTM for regression consists of five layers, i.e., input layer, LSTM layer, dropout layer, fully connected layer and regression output layer [30]. The basic functions of LSTM can be formulated as: 


$$
\left\{\begin{array}{l}
i_{t}=\operatorname{sigm}\left(W_{x i} x_{t}+W_{h i} h_{t-1}+b_{i}\right) \\
f_{t}=\operatorname{sigm}\left(W_{x f} x_{t}+W_{h f} h_{t-1}+b_{f}\right) \\
o_{t}=\operatorname{sigm}\left(W_{x o} x_{t}+W_{h o} h_{t-1}+b_{o}\right) \\
C_{t}=f_{t} \odot C_{t-1}+i_{t} \odot \tanh \left(W_{x c} x_{t}+W_{h c} h_{t-1}+b_{c}\right) \\
h_{t}=o_{t} \odot \tanh \left(C_{t}\right)
\end{array}\right.
$$

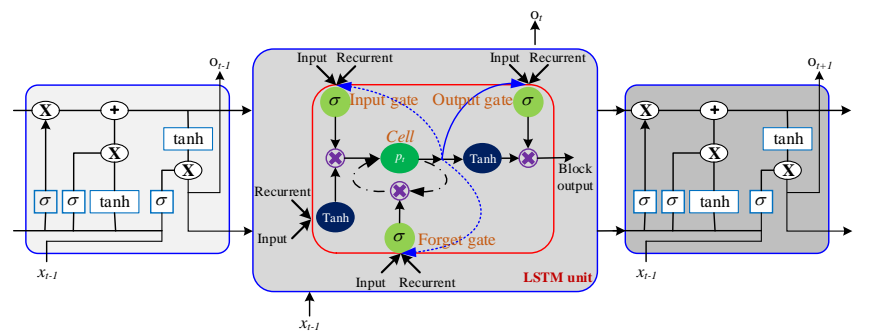

Fig. 1. Schematic diagram of LSTM.

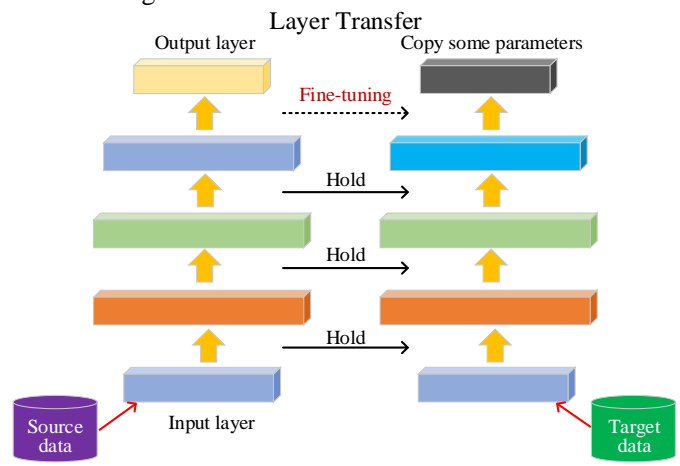

Fig. 2. Schematic diagram of transfer learning.

where $i, f, o, C$ and $h$ denote input gate, forget gate, output gate, state cell and output data, respectively; $b$. is the bias parameter; $W_{x}$. and $W_{h}$. respectively represent the weight matrices for input and previous output; $\odot$ indicates the element-wise product; sigm expresses the sigmoid function that is an activation function; tanh is defined as the hyperbolic function. In (1), the information amount for the cell memory to update, forget and output its state is determined by the first three equations, and the state cell and output are determined by the last two recursive equations. Herein, the root-mean-square error (RMSE) is applied to construct the loss function, as:

$$
R M S E=\sqrt{\frac{1}{N} \sum_{i=1}^{N}\left(S_{S O H}-S O H_{P r e}\right)^{2}}
$$

where $\mathrm{SOH}_{\mathrm{Ref}}$ and $\mathrm{SOH}_{\mathrm{Pre}}$ denote the reference value and the predicted value of $\mathrm{SOH}$, and $N$ means the data size of $\mathrm{SOH}$.

\section{B. Transfer Learning}

Existing machine learning methods usually assume that the data obey the same distribution, and also a large amount of labeled data are required to train the model. However, in real applications, the domain of the source dataset and target dataset are usually different, and the limited label data in the target data hindered the promotion of machine learning methods. To recognize the knowledge and skills learned from previous tasks and transfer them to novel domains/tasks, the TL based on deep neural networks is proposed and has attracted wide attention to now [31]. As an efficient data mining framework, TL can transfer the data or features from previous tasks (named as source domain hereinafter) to facilitate the model construction of target task (called as target domain), thus remedying the difficulty of information loss caused by the data insufficiency. For compensating the data loss on degradation prediction and improve the $\mathrm{SOH}$ estimation precision, a flexibility scheme for $\mathrm{SOH}$ prediction of different types of batteries is presented by integrating LSTM and TL. Among all TL solutions, fine-tuning of a pre-trained model based on a new dataset is the most popular manner for knowledge transfer in the machine learning field [32]. On this account, it is engaged in $\mathrm{SOH}$ estimation in this study, and the schematic diagram of TL with fine-tuning strategy is exhibited in Fig. 2.

The network consists of five layers, where the first layer is the input layer, and the last layer is the output layer. The input on the left and right networks includes respectively the source data and target data. Firstly, the network is pre-trained through the source data to obtain the base model. Then, the target data is set as the input variable, and the TL with fine-tuning strategy is activated to adjust the parameters of one middle layer, while the parameters of other layers remain unchanged. By this manner, a new transfer model is constructed. Since only partial parameters of the base model are adjusted, the training speed is much faster than that of retraining the whole model. Simultaneously, the proposed method can cooperatively transfer the cycle data and the formerly trained model of source battery to the newly built model of target batteries for easier $\mathrm{SOH}$ prediction. Its strong learning capability can bridge the serviceable information implied in the data and model between two domains to facilitate the prediction model construction of target domain, thus mitigating the requirement of aging data and obviously improving the training efficiency.

After detailing the principles of LSTM and TL, the related experiments and $\mathrm{SOH}$ prediction based on the proposed methods will be investigated.

\section{EXPERIMENTS AND FEATURE GENERATION}

This section details the aging experiment procedures and specifies the feature generation from the test data for $\mathrm{SOH}$ estimation.

\section{A. Aging Experiment}

In this study, three different sources of battery data are considered: 1 ) the nickel cobalt manganese (NCM) dataset from our laboratory; 2) the lithium cobalt oxide (LCO) dataset from the Center for Advanced Life Cycle Engineering (CALCE), which is an open-access depository supplied by the University of Maryland [33]; and 3) the commercial lithium iron phosphate (LFP) battery dataset from the Massachusetts Institute of Technology (MIT) and Stanford University [34]. These three datasets are named as dataset 1 , dataset 2 and dataset 3, respectively. The specifications of these three batteries are tabulated in Table I. The laboratory batteries are repeatedly experimented according to a constant current charge/discharge strategy at room temperature. During charging process, 2 A current is imposed in the constant current (CC) charging step, and $4.15 \mathrm{~V}$ is sustained in the constant voltage $(\mathrm{CV})$ charging 
stage until the current drops to the predefined cut-off value. Then, the test batteries are discharged with the $1 \mathrm{C}$ current, where $\mathrm{C}$ denotes the value of rated capacity with unit Amperehour (Ah), to the lower cut-off voltage after 5 minutes rest. The batteries in dataset 2 belong to commercial prismatic cells with 1100 milli Ah (mAh) nominal capacity. Three cells, referred to as 2-1, 2-2 and 2-3, are aged with the similar cycle experiment setting in dataset 1 , and the charging current is set to $0.55 \mathrm{~A}$. More detailed test steps can be found in [35]. For dataset 3, the charging protocol and chemistry property of batteries are different from the first two batteries. The cells are charged with a two-step fast-charging strategy: C1(Q1)-C2 scheme, in which $\mathrm{C} 1$ and $\mathrm{C} 2$ respectively indicate the first and second step current, and Q1 denotes the SOC at which the current switches from $\mathrm{C} 1$ to $\mathrm{C} 2$. The second step charge is terminated at $80 \%$ $\mathrm{SOC}$, followed by the $1 \mathrm{C}$ current based $\mathrm{CC}-\mathrm{CV}$ charge mode. Finally, $4 \mathrm{C}$ current is imposed to discharge the battery until its voltage reaches the minimum cut-off value [36]. The experimental data from the aging test are employed to extract battery health features, train the model and test the developed battery $\mathrm{SOH}$ estimation algorithm. Firstly, the extraction process of health features will be introduced.

\section{B. Feature Generation}

As discussed above, it is critical to find a representative health feature to properly assess the aging state. The health feature needs to be easy to obtain, simple to calculate, and efficient for anti-interference. In EV applications, since driving conditions and driving demands are time-varying and stochastic, the discharging process of lithium-ion batteries is usually stochastic and unforeseeable [36], and it is tricky to apply the discharge data for SOH estimation. Nonetheless, the charging process is generally uniform as the battery is usually charged from the grid according to specific instructions. Hence, the charging voltage and capacity profiles are designated for feature generation and $\mathrm{SOH}$ prediction. Taking the dataset 1 as an example, the charging voltage profiles under different cycles are plotted in Fig. 3 (a), and we can find that the charging time decreases with the increase of cycle numbers. Intuitively, the charging time for a fixed voltage range will be shortened when the battery capacity degrades. On this account, the duration for a fixed voltage range can be deemed as a health feature to quantify $\mathrm{SOH}$ variation. A full charging voltage profile under the CC scheme is presented in Fig. 3 (b), where $t_{1}$ and $t_{2}$ denote the moment when the voltage reaches the preset low and high threshold during the battery charging process, and $X$ indicates the feature vector. According to Fig. 3 (b), the detail feature generation procedures can be summarized, as follows:

1) Execute the pre-defined $\mathrm{CC}$ charge strategy for certain duration and measure the voltage throughout. Since a full charging cycle experiment from empty is difficult to acquire, a subset of the acquired points will be applied as the input representing the whole information of one cycle. As such, a definite voltage range based on the test data is marked, and the low and high boundaries are assumed as $V_{1}$ and $V_{2}$, respectively.

2) With the age of batteries, the polarization becomes severer with the form of internal resistance increase, and concretely, the $\mathrm{CC}$ charging time decreases. On this account, the charging time for a certain voltage range can be hired to measure the battery degradation. To be specific, for each charging curve, the duration $T_{i}$ in the designated voltage range, i.e., $V_{1}$ to $V_{2}$, is considered as a health feature, which can not only capture the degradation of capacity and increment of resistance, but also evaluate the inconsistency of battery cells in a pack to some extent.

In the next step, the $\mathrm{SOH}$ prediction scheme will be designed according to the proposed strategy and the identified healthy features.

\section{BATTERY PACK SOH PREDICTION SCHEME}

To avoid over operation of individual battery cell in packs, the minimum SOH value among that of cells is defined as the SOH of battery pack. Due to imperfect fabrication techniques in manufacturing process and inconsistent heat distribution inside battery pack, the aging speed of cells is different. In this section, a two-part SOH estimation model incorporating the CMM and CDM is developed, in which CMM describes the mean $\mathrm{SOH}$ performance among cells, and CDM mainly accounts for the difference between cell $\mathrm{SOH}$ and the mean value.

\section{A. Construction of Cell Mean Model}

The constructed block diagram of the CMM is depicted in Fig. 4. Firstly, the health features are extracted by the method defined in Section III, and then the basic model is built by the LSTM method to map the relationship between health features and SOH. Finally, the TL method with fine-tuning strategy is exerted to establish the autonomous learning model, and attain $\mathrm{SOH}$ prediction based on the reduced training data and mutative feature range. The whole prediction scheme mainly includes three steps:

1) Feature extraction: Partial voltage data are acquired from the voltage curves during $\mathrm{CC}$ charging process to generate the first health feature coefficient, and the specified charging interval for the preset voltage range is calculated as the second health feature factor. Both factors are selected as the input of the base model.

TABLE I. SPECIFICATIONS OF THE TEST BATTERIES.

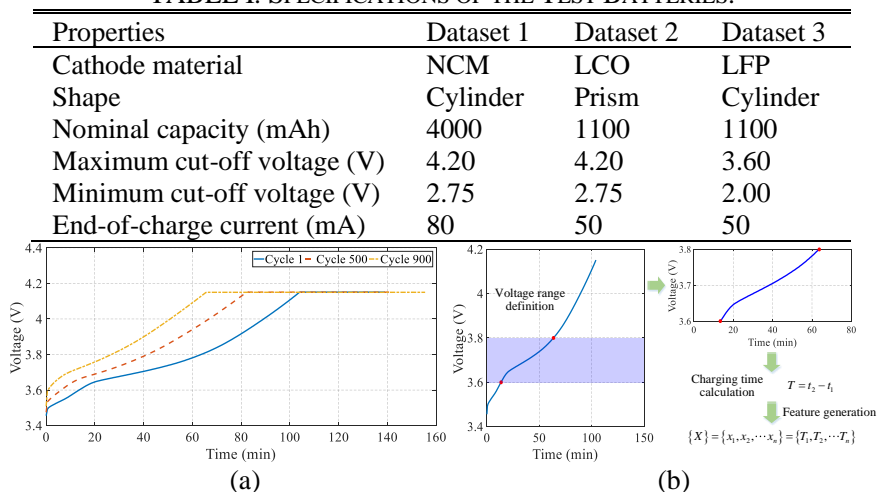

Fig. 3. Illustration of health feature generation. (a) $\mathrm{CC}-\mathrm{CV}$ charging profiles under different cycles; (b) Health feature extraction process. 


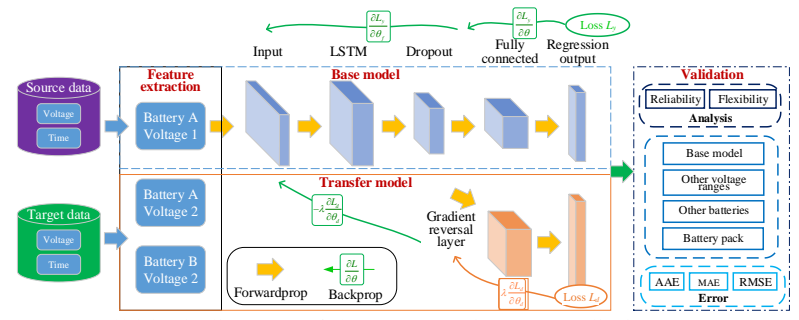

Fig. 4. Overall block diagram of the presented $\mathrm{SOH}$ prediction.

2) Base model construction: According to the offline test results, partial measurement data are selected as the training dataset, and the remaining data are assigned as the validation object. The ratio of partitioning the data between training and testing will be discussed in the next section. Then, the LSTM network with five layers is implemented to construct the base model. As well known, increasing layers will complicate the calculation process and aggravate the computation intensity. However, when the number of layers is too small, the model accuracy will be deteriorated. Through optimization, the number of layers is set to five after trading off the prediction accuracy and calculation complexity. It is known that environment temperature and charging/discharging current can generate significant influences on aging of lithium-ion batteries; however, it is quite difficult to fully account for the massive influences incurred by different operation conditions in long-term prediction of SOH. Hence, in the proposed scheme, a one-step-ahead prediction method is exploited to predict battery $\mathrm{SOH}$. That is, no matter how much the battery degrades, it is not necessary to know the previous capacity; and only if the specific characteristic parameter, i.e., health feature is extracted, the trained model can be employed to predict battery $\mathrm{SOH}$ efficiently. Additionally, although the current health feature is extracted to estimate the $\mathrm{SOH}$, the long-term dependence during SOH estimation is merged due to the longterm memory characteristics of LSTM algorithm. By this manner, the up-to-date aging state of lithium-ion batteries can be estimated comprehensively, and the impact of working environment on battery degradation can be considered to some extent.

3) Transfer model construction: To ensure the flexibility of the proposed $\mathrm{SOH}$ prediction method, the TL with fine-tuning strategy is exploited to adjust the parameters of the base model. In this scheme, only the parameters of the bottom two layers, i.e., the fully connected layer and regression output layer, will be updated, and the parameters of other layers will remain unchanged. Note that as the model parameters need to be adjusted according to the new features or battery types, the labeled data need to be prepared for retraining. By this manner, the transfer model is constructed.

\section{B. Construction of Cell Difference Model}

Here, we assume the $\mathrm{SOH}$ of the first cell in pack as the $\mathrm{CMM}$, the feature difference $\Delta T^{i}$ and the $\mathrm{SOH}$ difference $\triangle S O H^{i}$ between the mean value $\mathrm{SOH}_{\text {mean }}$ and $\mathrm{SOH}^{i}$ of $i$ th cell can be formulated as:

$$
\begin{gathered}
\Delta T^{i}=T^{i}-T_{\text {mean }} \\
\Delta S O H^{i}=S O H^{i}-S O H_{\text {mean }}
\end{gathered}
$$

where $T^{i}$ denotes the duration in the designated voltage range of cell $i ; T_{\text {mean }}$ indicates the duration in the designated voltage range of mean cell $\mathrm{SOH} . \triangle \mathrm{SOH}$ is negative for lower $\mathrm{SOH}^{i}$ and positive for higher $\mathrm{SOH}^{i}$, compared to the mean value. To map the hidden relationship between $\Delta T^{i}$ and $\triangle S O H^{i}$, LSTM is employed to establish the CDM, in which $\Delta T^{i}$ and $\triangle S O H^{i}$ are assigned as the input and output. After attaining the mean value and the difference, the minimum difference under the same cycle is selected. Then, the minimum cell $\mathrm{SOH}$, i.e., the pack $\mathrm{SOH}$, can be deduced by inverse transformation of (3). During the estimation process, only the duration for a preset voltage range needs to be determined online, and it is easy to acquire. Meanwhile, the real capacity or SOH of the training set needs to be known in advance for model training, and the real capacity of the test set is treated as the reference to evaluate the model performance.

By this manner, the SOH inconsistency within the battery pack can be well evaluated by the proposed CDM, and the proposed method can effectively distinguish the cell inconsistency and identify the battery pack SOH state. Next, the experimental validation will be conducted and discussed.

\section{VALIDATION AND ANALYSIS}

The performance of the proposed scheme for $\mathrm{SOH}$ prediction is evaluated by conducting a large number of comparisons raised by different predictors under various operation conditions. To demonstrate the feasibility and advancement of the proposed method, four representative tests are conducted, including the performance comparison of different predictors in the base model with training and testing datasets of different length, the portability over other voltage ranges and battery types as well as validations on battery packs. The evaluation criteria include average absolute error (AAE), maximum absolute error (MAE) and RMSE, the AAE and MAE are formulated as:

$$
\left\{\begin{array}{l}
A A E=\frac{1}{N} \sum_{i=1}^{N}\left|S O H_{R e f}-S O H_{\text {Pre }}\right| \\
M A E=\operatorname{Max}\left(\left|S O H_{\text {Ref }}-S O H_{\text {Pre }}\right|\right)
\end{array}\right.
$$

\section{A. Comparison of Different Methods on Base Model}

Based on the experimental data, the feasibility of the proposed base model is firstly validated in terms of prediction of dataset 1. Another three commonly used $\mathrm{SOH}$ predictors, including back propagation NN (BP-NN), SVM and least square SVM (LS-SVM), are employed to conduct the estimation for performance comparison. The influence of voltage range selection is also addressed in this part. In the three commonly used predictors, the voltage range of $3.6 \mathrm{~V}$ to $3.8 \mathrm{~V}$ is applied to extract the health feature. In the proposed method, three different ranges are considered for healthy feature generation. The first two ranges express individual voltage interval, and the third one includes two ranges, as presented in Table II. For each prediction scenario, the first $70 \%$ of aging profiles are applied for model training, and all the four methods 
are applied to forecast the same degradation data. Moreover, to fully promote the advantages of each network, the grid search method is leveraged to find the optimal parameters of each model.

The prediction performance of these four methods is compared with the reference curve, and the results are shown in Fig. 5 and Table II, respectively. As can be found, the capacity degradation trend is generally consistent, and no obvious rapid aging process emerges. Note that as the phenomenon of capacity recovery exists due to the intermittent experiment, the capacity degradation curve is not monotonously decreasing. From Fig. 5 and Table II, it can be observed that all the four approaches can effectively track the reference curve during the entire lifetime, the AAEs by the BP-NN, SVM and LS-SVM are $0.43 \%, 0.50 \%$ and $0.43 \%$, respectively; in contrast, the largest AAE for the proposed scheme is less than $0.43 \%$. After removing the estimation results in the initial stage, the MAE of the proposed method is much lower than that of other three algorithms, except when two voltage ranges are selected to extract the health feature. Additionally, for the BP-NN, SVM and LS-SVM algorithms, their RMSEs are respectively $0.63 \%$, $0.69 \%$ and $0.60 \%$, obviously higher than that of the proposed method. The running time of the proposed method is evaluated on a desktop computer, which is equipped with Intel Xeon E3$1230(3.30 \mathrm{GHz})$ processor and $32 \mathrm{~GB}$ memory. The calculation time of the proposed method is $3.02 \mathrm{~s}$ when using dataset 1 to predict $\mathrm{SOH}$. Since $\mathrm{SOH}$ can be merely estimated once per cycle, the average running time of each cycle for dataset 1 is $3.34 \mathrm{~ms}$. Compared with the duration of one cycle experiment that lasts for a number of hours, the short estimation duration can fully meet the requirements of online implementation. By comparing the prediction results, it can be concluded that different voltage ranges as health features lead to different estimation performance. It is also distinct that the developed method with the input data under range from $3.6 \mathrm{~V}$ to $3.8 \mathrm{~V}$ can raise better prediction performance, compared to that with other voltage ranges. Additionally, the model inputs with two voltage ranges will possibly increase the model instability and consequent computational complexity. Therefore, we can infer that the proposed algorithm with five layers and the health feature extracted from a single voltage range are feasible and reasonable.

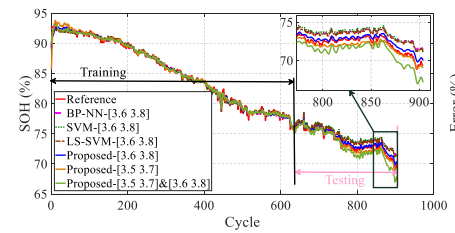

(a)

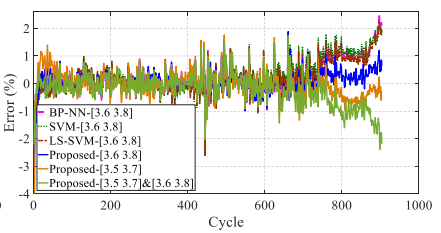

(b)
Fig. 5. Prediction results of different methods on base model. (a) SOH curves; (b) Error curves.

TABLE II. Comparison Results of Different Methods.

\begin{tabular}{lllll}
\hline \hline Algorithm & Voltage range & AAE $(\%)$ & MAE $(\%)$ & RMSE (\%) \\
\hline BP-NN & {$\left[\begin{array}{lll}3.6 & 3.8\end{array}\right]$} & 0.43 & 2.56 & 0.63 \\
SVM & {$\left[\begin{array}{lll}3.6 & 3.8\end{array}\right]$} & 0.50 & 2.21 & 0.69 \\
LS-SVM & {$\left[\begin{array}{lll}3.6 & 3.8\end{array}\right]$} & 0.43 & 2.63 & 0.60 \\
& {$\left[\begin{array}{lll}3 & 6 & 3.8\end{array}\right]$} & 0.31 & 1.88 & 0.42 \\
Proposed & {$\left[\begin{array}{lll}3.5 & 3.7\end{array}\right]$} & 0.36 & 1.81 & 0.54 \\
& {$\left[\begin{array}{lll}3.5 & 3.7\end{array}\right] \&$} & 0.42 & 2.38 & 0.59 \\
\hline
\end{tabular}

\section{B. Performance Evaluation on Different Voltage Ranges}

In previous discussions, a random voltage range is selected to construct the base model. To validate the adaptability of the proposed scheme, the voltage range for health feature extraction is modified to 3.5 to $4 \mathrm{~V}$ and 3.5 to $4.15 \mathrm{~V}$, significantly deviating from the preset voltage range of the base model. Fig. 6 displays the results for different voltage ranges by the proposed method as well as the single LSTM method. In this case, the model trained by the first 360 cycle data (40\% of the experimental data) are utilized to predict the $\mathrm{SOH}$ of the remaining 544 cycles. From Fig. 6, it can be found that the predicted $\mathrm{SOH}$ is in general less accurate than before, and the MAE becomes larger, due to the improper selection of the voltage range and the amount decrease of the training dataset. Even so, the estimation performance based on the proposed method is still acceptable. However, the SOH curves obtained by the proposed method and the single LSTM method show different trends, and the prediction profile of the former method can track the degradation curve more accurately during the whole process; nonetheless, the latter only follows the reference curve during training and gradually moves away from the true value during test.

Similar as before, the proposed approach achieves more robust results with less training data and varying feature range. Fig. 6 (c) shows the boxplot of the prediction errors across all the voltage ranges based on the proposed and individual LSTM methods, and the red lines represent the median value of error. We can find that the boxplots of LSTM and TL-based method for different voltage ranges are flatter, show that the error distribution of this method is more concentrated. Moreover, the median error line based on the LSTM and TL method is closer to zero. The maximum error of the proposed method occurs in the initial cycle, which is included in the training set. However, the maximum error of individual LSTM method appears in the test set and higher than $9 \%$. It can be concluded that the proposed scheme outperforms than the single LSTM approach. Moreover, it also can be observed from the above analysis that the proposed method can result in precise $\mathrm{SOH}$ estimation with the MAE of $3.2 \%$. Moreover, this method can reduce the training data amount by about $30 \%$ (about 270 cycles). From the perspective of balancing data preparation and accuracy, it is still acceptable to sacrifice the slight accuracy while reducing a variety of test data and saving much testing time.

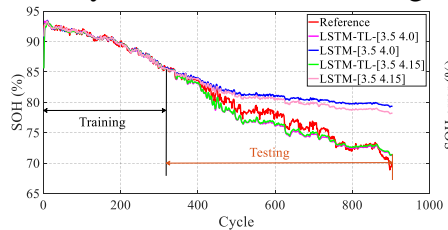

(a)

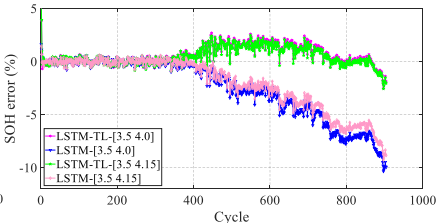

(b)

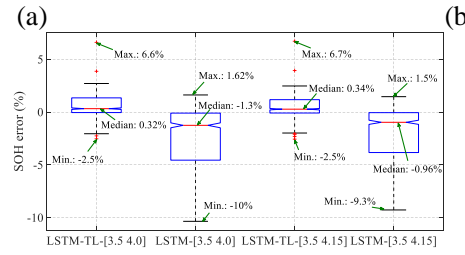

(c) 
Fig. 6. Prediction results of different voltage ranges. (a) Comparison of $\mathrm{SOH}$ prediction; (b) Comparison of $\mathrm{SOH}$ error; (c) Boxplot of different voltage ranges.

\section{Performance Evaluation on Other Batteries}

To verify the adaptability and scalability of the proposed scheme to other types of batteries. The datasets 2 and 3 specified in Table I are applied to estimate the $\mathrm{SOH}$ via the proposed method, and the performance of dataset 2 is presented in Fig. 7. Compared with dataset 1, the capacity recovery phenomenon of dataset 2 is more obvious. With the help of TL with fine-tuning strategy, the model is trained by the data of the first 100 cycles (occupying $18 \%$ to $20 \%$ of the aging data), which is determined through trial and error. The remaining cycles are applied for validation, and the selected voltage interval for feature generation ranges from $3.8 \mathrm{~V}$ to $4.1 \mathrm{~V}$. Although only 100 cycles data are leveraged to train the model, the developed method leads to satisfactory performance during the entire aging process. In the worst case, the maximum AAE, MAE and RMSE are $0.82 \%, 3.9 \%$ and $1.07 \%$, respectively. Note that the MAEs of the cells 2-1 and 2-2 exceed 3\%, and it can be seen from Fig. 7 (b), (e) and (h) that most of the errors can be restricted within $2.8 \%$. Concretely, the error distribution can be schematically summarized in Fig. 7 (c), (f) and (i). As can be found, the zero (or near) error emerges frequently. With the increase of the absolute value of error, the error occurrence probability decreases gradually, being in line with the Gaussian distribution with the mean value of $0.04 \%, 0.3 \%, 0.006 \%$ and the variance of $0.0002,0.00008,0.00006$. The detailed validation results reveal that the distribution of error is reasonable, and manifest that the proposed method can accurately and stably estimate battery $\mathrm{SOH}$, even in the case of obvious size reduction of training dataset. Moreover, the proposed method can effectively reduce the degradation experiment by 285 cycles, which will cost around 855 hours in this case. This in turn highlights the strong timeliness and high efficiency of the proposed framework when it is leveraged to predict the $\mathrm{SOH}$ of different batteries, manifesting its adaptability and extendibility.

After the comparison based on dataset 2, dataset 3 is further experimented to examine the performance of the proposed method. The cathode material of dataset 3 is LFP, which is different from the first two types of batteries. Here, only one cell is chosen for validation, and the voltage range of $2.5 \mathrm{~V}$ to $3.5 \mathrm{~V}$ is selected as the health feature. The prediction results are sketched in Fig. 8, from which we can find that the general degradation trend looks like a polynomial curve, and hence an efficient polynomial fitting method may gain preferable estimation with the input of cycle number. However, from the zoomed-in figure in Fig. 8 (a), local partial capacity recovery emerges, making it difficult to accurately estimate the $\mathrm{SOH}$ by the simple polynomial fitting method. On the other hand, since the battery in dataset 3 is fully charged and discharged during experiment, the $\mathrm{SOH}$ value with respect to cycle number is close to a polynomial curve. Nevertheless, it is difficult to encounter full charge and discharge operations all the time in practice. Once partial charge and discharge behaviors occur, the number of cycles will increase, while the $\mathrm{SOH}$ degradation rate will be slower, and in this case the polynomial curve fitting method cannot work well. Additionally, as the research target of this paper is to investigate a general efficient $\mathrm{SOH}$ prediction algorithm that can adapt to different types of batteries with the reduction of training data size, it is obvious that the polynomial fitting method and the related settings based on dataset 3 are not applicable for datasets 1 and 2. Instead, the LSTM network and TL with fine-tuning strategy based on dataset 1 can be transplanted to predict $\mathrm{SOH}$ of dataset 3 with preferable and robust estimation. In addition, the single LSTM algorithm is continuously exploited to compare the size of training data. In the two networks, except the training data, the other parameters remain the same. Through repetitive optimization, the first $80 \%$ (1076 cycles) and 60\% (611 cycles) of aging data are respectively applied for training the single LSTM and the present algorithm, and all the data are exerted to assess the prediction performance. As can be found, the prediction results do not show distinct difference, and both methods can provide precise predictions. Concretely, the RMSE and AAE by the LSTM are $0.27 \%$ and $0.16 \%$, and those by the proposed method remain close and are $0.36 \%$ and $0.24 \%$. Nonetheless, the MAE by the proposed method is $0.09 \%$, much lower than that by the LSTM; and moreover, the dataset size for training the proposed method is $20 \%$ less than that for training the single LSTM algorithm. In other words, with the same estimation results, 465 cycle tests can be saved by the proposed method in this study, and most of the estimation error can still be controlled within $2.8 \%$, which is only $0.92 \%$ higher than that of the base model. Thus, it can be summarized that the proposed algorithm can be transplanted from NCM batteries to LCO and LFP batteries, manifesting its superior extendable capability. The only task that needs to be conducted is to adjust the parameters of the fully connected layer and output layer. By this manner, the feasibility of the proposed algorithm when applied in different types of batteries is verified.

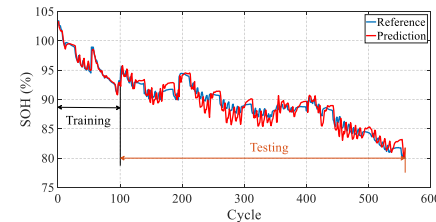

(a)

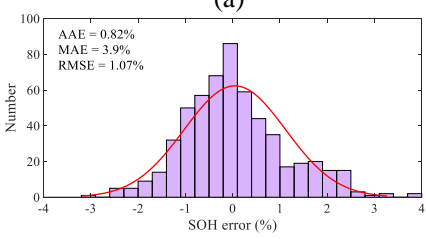

(c)

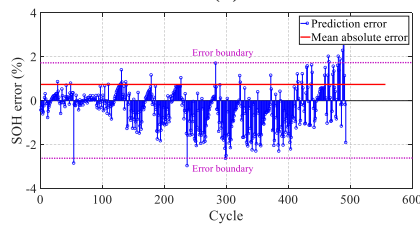

(e)

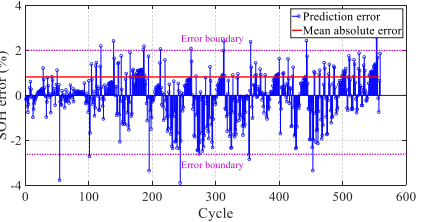

(b)

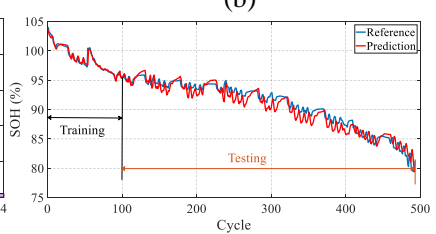

(d)

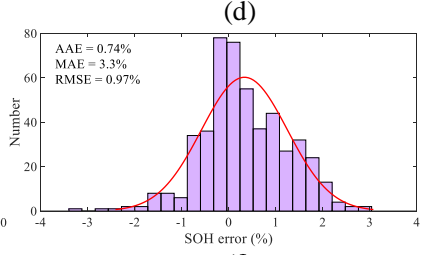

(f) 


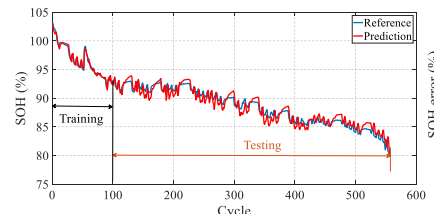

(g)

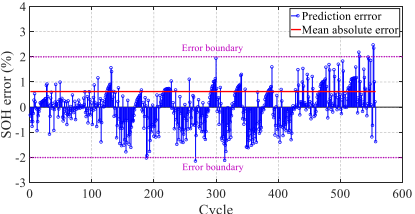

(h)

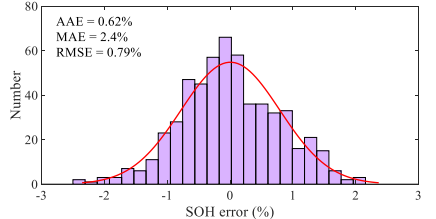

(i)

Fig. 7. Prediction results for the dataset 2. (a) - (c) SOH prediction curves, errors and errors histogram distribution of cell 2-1; (d) - (f) $\mathrm{SOH}$ prediction curves, errors and errors histogram distribution of cell 2-2; (g) - (h) $\mathrm{SOH}$ prediction curves, errors and errors histogram distribution of cell 2-3.

\section{Performance Evaluation on Battery Pack}

Four cells grouped into a small pack by serial connection are experimented with the same environmental conditions. These four cells are with the same property in dataset 1 , and referred to as cell 1-1, 1-2, 1-3 and 1-4, respectively. The acquired data are utilized to identify the $\mathrm{SOH}$ by the proposed pack estimation algorithm. In this study, the estimation results of cell 1-1 are hired to construct the CMM. Note that the validation in Part A is based on the experiment of cell 1-1, and hence the estimation results of cell 1-1 are not repeated here. The estimation curves of CDM for cells 1-2, 1-3 and 1-4 are depicted in Fig. 9 (a), (d) and $(\mathrm{g})$. It can be clearly found the evolution trend of CDM for each cell shows different trend. In the first 200 cycles, the differences are not obvious, and the maximum $\mathrm{SOH}$ difference is less than $2 \%$. However, as the battery ages, the difference of CDM becomes more and more obvious; and when the cycle reaches 440 times, the maximum difference exceeds 3\%.

The prediction results of cell $\mathrm{SOH}$ are sketched in Fig. 9 (b), (e) and (h), and their corresponding errors are plotted in Fig. 9 (c), (f) and (i) and summarized in Table III, where "LSTM" means the SOH obtained by the single LSTM algorithm. As can be seen, the prediction curves of the proposed method are closer to the reference profiles than those by the single LSTM. The prediction error of the proposed method for all the cells is located closer to the horizontal axis. Also, the result of MAE is better than that of the single LSTM method, as portrayed in Fig. 9 (c), (f) and (i). Moreover, due to the long-term memory characteristics of single LSTM, the initial state of LSTM fluctuates obviously, raising larger $\mathrm{SOH}$ estimation error. By contrast, thanks to the LSTM-TL method and CDM, the SOH prediction results by the proposed method do not show the similar phenomenon.

The diagnosis results of battery pack SOH for single LSTM and the proposed method are shown in Fig. 10, and the statistical error is listed in Table III. As can be seen, the prediction value of the single LSTM is mostly below the reference value, while the estimation value of the proposed method oscillates around the reference. Obviously, the proposed method enables more reasonable variation track of pack SOH, compared to the single LSTM method. According to Table III, the evaluation indexes reveal that the proposed method performs more accurate than single LSTM. To be specific, the AAE, MAE and RMSE of the proposed method are less than two thirds, one third and three fifths of that by the single LSTM method.

To sum up, the proposed LSTM integrating TL algorithm and CDM can achieve satisfactory pack SOH estimation. As such, the abuse of battery packs can be effectively avoided by reliably diagnosing the pack $\mathrm{SOH}$ and safe operation of batteries can be guaranteed. Moreover, the prediction procedure of the proposed scheme is executed during the charging phase, indicating its easy application potential in practice. Additionally, in the proposed pack SOH prediction scheme, the LSTM model is employed as the CDM, and the minimum estimation value of $\mathrm{CDM}$ is identified to determine the pack $\mathrm{SOH}$. When facing the $\mathrm{SOH}$ of a battery pack with multiple cells (for example tens to thousands), firstly the CDM value of each cell needs to be compared, and the minimum CDM will be selected. Then, the estimation method will be exploited to predict the entire battery pack SOH. From this point of view, applying the proposed scheme to the pack with multiple cells increases only the amount of CDM comparison, and thus the estimation performance of pack $\mathrm{SOH}$ by the proposed algorithm will be still the same as addressed in this study.

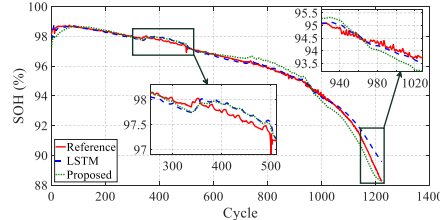

(a)

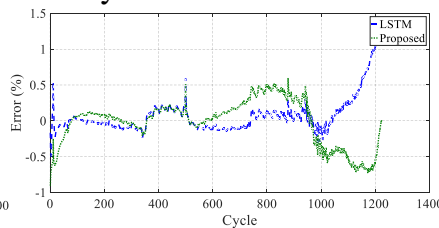

(b)
Fig. 8. Prediction results for the dataset 3. (a) SOH prediction curve; (b) $\mathrm{SOH}$ prediction error.

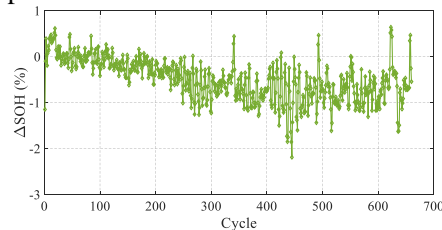

(a)

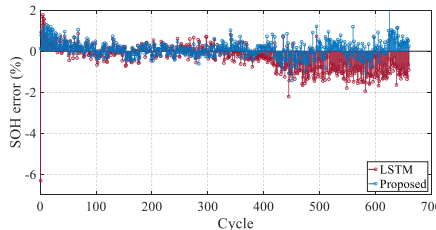

(c)

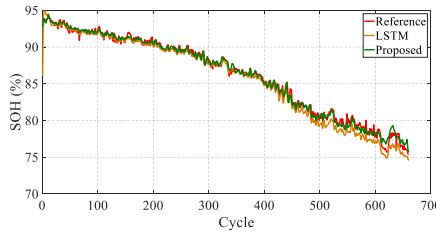

(e)

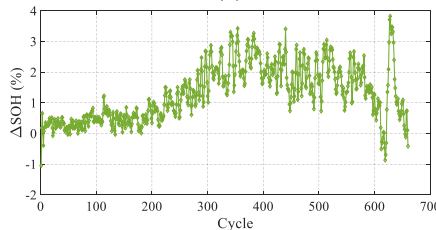

(g)

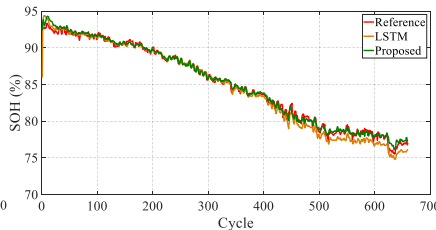

(b)

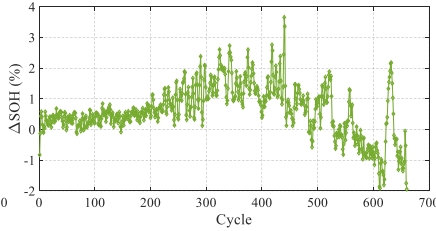

(d)

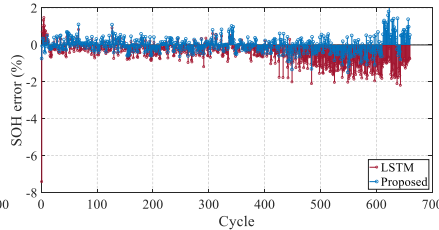

(f)

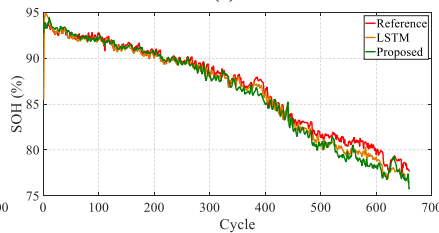

(h) 


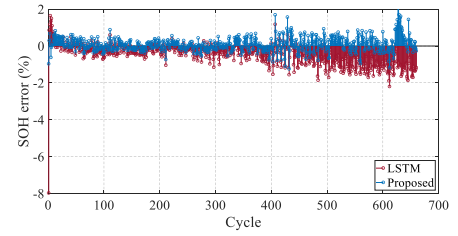

(i)

Fig. 9. SOH prediction results for battery cells. (a) - (c) CDM curve, $\mathrm{SOH}$ evolution curves and errors for cell 1-2; (d) - (f) CDM curve, SOH evolution curves and errors for cell 1-3; (g) - (i) CDM curve, SOH evolution curves and errors for cell 1-4.

TABLE III. COMPARISON RESULTS OF BATTERY PACK.

\begin{tabular}{|c|c|c|}
\hline Battery & Method & AAE (\% \\
\hline \multirow{2}{*}{ Cell 1-2 } & LSTM & 0.39 \\
\hline & Proposed & 0.31 \\
\hline \multirow{2}{*}{ Cell 1-3 } & LSTM & 0.45 \\
\hline & Proposed & 0.31 \\
\hline \multirow{2}{*}{ Cell 1-4 } & LSTM & 0.41 \\
\hline & Proposed & 0.30 \\
\hline \multirow{2}{*}{ Pack } & LSTM & 0.51 \\
\hline & Proposed & 0.31 \\
\hline & & $\begin{array}{l}\text { Referencec } \\
\text { LSTM } \\
\text { - Proposed }\end{array}$ \\
\hline & & 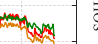 \\
\hline 100 & 500 & 700 \\
\hline
\end{tabular}

(a)

\begin{tabular}{ll} 
MAE $(\%)$ & RMSE $(\%)$ \\
\hline 6.31 & 0.63 \\
2.01 & 0.41 \\
7.41 & 0.71 \\
2.01 & 0.43 \\
7.95 & 0.67 \\
2.35 & 0.42 \\
6.80 & 0.71 \\
1.82 & 0.42
\end{tabular}

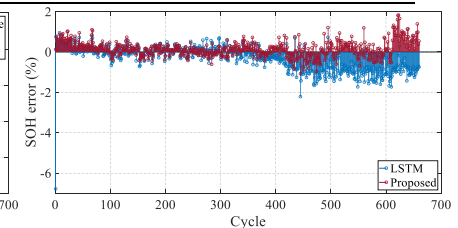

(b)

Fig. 10. SOH prediction results for lithium-ion battery pack. (a) SOH evolution for battery pack; (b) SOH errors for battery pack.

\section{CONCLUSIONS}

The knowledge of battery pack $\mathrm{SOH}$ is of vital importance for safe operation. Machine learning-based methods require a large amount of training data to portray the hidden nonlinear relationship of lithium-ion batteries to predict $\mathrm{SOH}$. In this study, a flexible LSTM based battery pack SOH prediction scheme is advanced with the combination of TL strategy. The relative charging time for a pre-set voltage range is extracted as the health feature to represent the degradation state of lithiumion battery cells. Then, an improved LSTM network integrating $\mathrm{TL}$ is proposed to establish the CMM and fully substantiated by comparing with the commonly used $\mathrm{SOH}$ prediction algorithms. The validation results based on the proposed model manifest that the cell $\mathrm{SOH}$ error is less than 3\% with $70 \%$ training data. Moreover, the flexibility of the proposed TL algorithm is validated on different voltage ranges and different types of batteries, and the training data can be reduced by $20 \%$ to $40 \%$ without discrediting the estimation performance, thereby justifying the remarkable contribution of TL in mitigating the training data amount. Based on the developed $\mathrm{CDM}$, the SOH inconsistency existing in battery cells can be effectively taken into account, and the pack $\mathrm{SOH}$ is properly determined by the CMM and minimum value of CDM. According to the proposed cell and pack $\mathrm{SOH}$ prediction methods, the pack SOH can reasonably follow the variation trend of the minimum $\mathrm{SOH}$ of cells, and therefore enables the avoidance of improper abuse operations more efficiently.

Although a smaller power battery pack is employed as the validation object in this study, the proposed scheme is also feasible to predict $\mathrm{SOH}$ of larger battery packs, which will be our validation focus in the next step. In the future, the aging mechanism and $\mathrm{SOH}$ diagnosis of lithium-ion batteries from the perspective of electrochemical reactions and the long-term prediction of battery $\mathrm{SOH}$ are imperative to be explored for promotion of estimation precision. In addition, the impact of temperature on performance variation and $\mathrm{SOH}$ prediction of lithium-ion batteries also needs to be further investigated.

\section{REFERENCES}

[1] N. Guo, X. Zhang, Y. Zou, B. Lenzo, and T. Zhang, "A Computationally Efficient Path-Following Control Strategy of Autonomous Electric Vehicles With Yaw Motion Stabilization," IEEE Trans. Transport. Electrific., vol. 6, no. 2, pp. 728-739, 2020, doi: 10.1109/TTE.2020.2993862.

[2] X. Hu, C. Zou, X. Tang, T. Liu, and L. Hu, "Cost-Optimal Energy Management of Hybrid Electric Vehicles Using Fuel Cell/Battery Health-Aware Predictive Control," IEEE. T. Power Electr., vol. 35, no. 1, pp. 382-392, 2020, doi: 10.1109/TPEL.2019.2915675.

[3] X. Shu, G. Li, J. Shen, W. Yan, Z. Chen, and Y. Liu, "An adaptive fusion estimation algorithm for state of charge of lithium-ion batteries considering wide operating temperature and degradation," J. Power Sources, vol. 462, p. 228132, 2020.

[4] X. Ding; D. Zhang; J. Cheng; B. Wang; Y. Chai; Z. Zhao, R. Xiong, and P. Luk, "A novel active equalization topology for series-connected lithium-ion battery packs," IEEE Transactions on Industry Applications, vol. 56, no. 6, pp. 6892-6903, 2020.

[5] Z. Deng, X. Hu, X. Lin, Y. Che, L. Xu, and W. Guo, "Data-driven state of charge estimation for lithium-ion battery packs based on Gaussian process regression," Energy, vol. 205, p. 118000, 2020/08/15/2020, doi: https://doi.org/10.1016/j.energy.2020.118000.

[6] X. Hu, L. Xu, X. Lin, and M. Pecht, "Battery lifetime prognostics," Joule, vol. 4, no. 2, pp. 310-346, 2020.

[7] X. Shu, G. Li, J. Shen, Z. Lei, Z. Chen, and Y. Liu, "A uniform estimation framework for state of health of lithium-ion batteries considering feature extraction and parameters optimization," Energy, vol. 204, p. 117957, 2020/08/01/ 2020, doi: https://doi.org/10.1016/j.energy.2020.117957.

[8] Z. Deng, X. Hu, X. Lin, L. Xu, Y. Che, and L. Hu, "General Discharge Voltage Information Enabled Health Evaluation for Lithium-Ion Batteries," IEEE/ASME T. Mech., pp. 1-1, 2020, doi: 10.1109/TMECH.2020.3040010.

[9] X. Shu, G. Li, Y. Zhang, J. Shen, Z. Chen, and Y. Liu, "Online diagnosis of state of health for lithium-ion batteries based on short-term charging profiles," J. Power Sources, vol. 471, p. 228478, 2020.

[10] Y. Tan and G. Zhao, "Transfer Learning With Long Short-Term Memory Network for State-of-Health Prediction of Lithium-Ion Batteries," IEEE Trans. Ind. Electron., vol. 67, no. 10, pp. 8723-8731, 2020, doi: 10.1109/TIE.2019.2946551.

[11] D. Xiao, G. Fang, S. Liu, S. Yuan, R. Ahmed, S. Habibi, and A. Emadi, "Reduced-Coupling Coestimation of SOC and SOH for Lithium-Ion Batteries Based on Convex Optimization," IEEE Trans. Power Electrific., vol. 35, no. 11, pp. 12332-12346, 2020, doi: 10.1109/TPEL.2020.2984248

[12] B. Duan, Q. Zhang, F. Geng, and C. Zhang, "Remaining useful life prediction of lithium - ion battery based on extended Kalman particle filter," International Journal of Energy Research, vol. 44, no. 3, pp. 1724-1734, 2020.

[13] X. Tang, Y. Wang, C. Zou, K. Yao, Y. Xia, and F. Gao, "A novel framework for Lithium-ion battery modeling considering uncertainties of temperature and aging," Energ. Convers. Manage., vol. 180, pp. 162$170,2019$.

[14] C. Chen, R. Xiong, and W. Shen, "A lithium-ion battery-in-the-loop approach to test and validate multiscale dual $\mathrm{H}$ infinity filters for stateof-charge and capacity estimation," IEEE Trans. Power Electron., vol. 33, no. 1, pp. 332-342, 2017.

[15] J. He, Z. Wei, X. Bian, and F. Yan, "State-of-Health Estimation of Lithium-Ion Batteries Using Incremental Capacity Analysis Based on Voltage-Capacity Model," IEEE Trans. Transport. Electrific., vol. 6, no. 2, pp. 417-426, 2020, doi: 10.1109/TTE.2020.2994543.

[16] X. Tang, K. Liu, X. Wang, F. Gao, J. Macro, and W. D. Widanage, "Model Migration Neural Network for Predicting Battery Aging Trajectories," IEEE Trans. Transport. Electrific., vol. 6, no. 2, pp. 363374, 2020, doi: 10.1109/TTE.2020.2979547. 
[17] J. Tian, R. Xiong, and W. Shen, "State-of-Health Estimation Based on Differential Temperature for Lithium Ion Batteries," IEEE Trans. Power Electron., vol. 35, no. 10, pp. 10363-10373, 2020, doi: 10.1109/TPEL.2020.2978493.

[18] X. Li, Z. Wang, and J. Yan, "Prognostic health condition for lithium battery using the partial incremental capacity and Gaussian process regression," J. Power Sources, vol. 421, pp. 56-67, 2019/05/01/ 2019, doi: https://doi.org/10.1016/j.jpowsour.2019.03.008.

[19] Y. Li, C. Zou, M. Berecibar, E. Nanini-Maury, J. C.-W. Chan, P. van den Bossche, J. Van Mierlo, and N. Omar, "Random forest regression for online capacity estimation of lithium-ion batteries, "Appl. Energ., vol. 232, pp. 197-210, 2018.

[20] C. She, Z. Wang, F. Sun, P. Liu, and L. Zhang, "Battery aging assessment for real-world electric buses based on incremental capacity analysis and radial basis function neural network," IEEE Trans. Ind. Informat., vol. 16, no. 5, pp. 3345-3354, 2019.

[21] P. Guo, Z. Cheng, and L. Yang, "A data-driven remaining capacity estimation approach for lithium-ion batteries based on charging health feature extraction," J. Power Sources, vol. 412, pp. 442-450, 2019.

[22] Y. Zhou, Y. Wang, K. Wang, L. Kang, F. Peng, L. Wang, and J. Pang, "Hybrid genetic algorithm method for efficient and robust evaluation of remaining useful life of supercapacitors," Appl. Energ., vol. 260, p. 114169, 2020.

[23] Y. Wu, Q. Xue, J. Shen, Z. Lei, Z. Chen, and Y. Liu, "State of Health Estimation for Lithium-Ion Batteries Based on Healthy Features and Long Short-Term Memory," IEEE Access, vol. 8, pp. 28533-28547, 2020.

[24] R. R. Richardson, C. R. Birkl, M. A. Osborne, and D. A. Howey, "Gaussian process regression for in situ capacity estimation of lithiumion batteries," IEEE Trans. Ind. Informat., vol. 15, no. 1, pp. 127-138, 2018.

[25] X. Hu, Y. Che, X. Lin, and Z. Deng, "Health Prognosis for Electric Vehicle Battery Packs: A Data-Driven Approach," IEEE/ASME T. Mech., pp. 1-1, 2020, doi: 10.1109/TMECH.2020.2986364.

[26] Y. Zheng, M. Ouyang, L. Lu, J. Li, X. Han, L. Xu, H. Ma, T. A. Dollmeyer, and V. Freyermuth, "Cell state-of-charge inconsistency estimation for $\mathrm{LiFePO} 4$ battery pack in hybrid electric vehicles using mean-difference model," Appl. Energ., vol. 111, pp. 571-580, 2013/11/01/ 2013.

[27] Y. Tian, R. Lai, X. Li, L. Xiang, and J. Tian, "A combined method for state-of-charge estimation for lithium-ion batteries using a long shortterm memory network and an adaptive cubature Kalman filter," Appl. Energ., vol. 265, p. 114789, 2020.

[28] J. Hong, Z. Wang, and Y. Yao, "Fault prognosis of battery system based on accurate voltage abnormity prognosis using long short-term memory neural networks," Appl. Energ., vol. 251, p. 113381, 2019/10/01/ 2019, doi: https://doi.org/10.1016/j.apenergy.2019.113381.

[29] X. Shu, G. Li, Y. Zhang, S. Shen, Z. Chen, and Y. Liu, "Stage of Charge Estimation of Lithium-ion Battery Packs Based on Improved Cubature Kalman Filter with Long Short-Term Memory Model," IEEE Trans. Transport. Electrific., pp. 1-1, 2020, doi: 10.1109/TTE.2020.3041757.

[30] Y. Zhang, R. Xiong, H. He, and M. G. Pecht, "Long short-term memory recurrent neural network for remaining useful life prediction of lithiumion batteries," IEEE Trans. Veh. Technol., vol. 67, no. 7, pp. 5695-5705, 2018.

[31] Y. Guo, H. Shi, A. Kumar, K. Grauman, T. Rosing, and R. Feris, "Spottune: transfer learning through adaptive fine-tuning," in Proceedings of the IEEE Conference on Computer Vision and Pattern Recognition, 2019, pp. 4805-4814.

[32] M. Long, Y. Cao, J. Wang, and M. Jordan, "Learning transferable features with deep adaptation networks," in International conference on machine learning, 2015: PMLR, pp. 97-105.

[33] Y. Xing, E. W. Ma, K.-L. Tsui, and M. Pecht, "An ensemble model for predicting the remaining useful performance of lithium-ion batteries," Microelectron. Reliab, vol. 53, no. 6, pp. 811-820, 2013.

[34] K. A. Severson, P. M. Attia, N. Jin, N. Perkins, B. Jiang, Z. Yang, M. H. Chen, M. Aykol, P. K. Herring, and D. Fraggedakis, "Data-driven prediction of battery cycle life before capacity degradation," Nature Energy, vol. 4, no. 5, pp. 383-391, 2019.

[35] W. He, N. Williard, M. Osterman, and M. Pecht, "Prognostics of lithium-ion batteries based on Dempster-Shafer theory and the Bayesian Monte Carlo method," J. Power Sources, vol. 196, no. 23, pp. 1031410321, 2011.
[36] X. Hu, Y. Che, X. Lin, and S. Onori, "Battery health prediction using fusion-based feature selection and machine learning," IEEE Trans. Transport. Electrific., pp. 1-1, 2020, doi: 10.1109/TTE.2020.3017090. 